ISSN 1997-5902

\title{
Sur l'origine fongique des galles observées chez les Eucalyptus
}

\author{
Anasse NOUNSI, Abdelaziz El ASRI, Amina OUAZZANI TOUHAMI, Rachid BENKIRANE et Allal \\ DOUIRA \\ Laboratoire de Botanique et de Protection des Plantes, UFR de Mycologie, Département de Biologie, Faculté des \\ Sciences, BP. 133, Université Ibn Tofail, Kénitra, Maroc.
}

Corresponding author email: douiraallal@hotmail.com

Original submitted in on $7^{\text {th }}$ December 2012. Published online at www.m.elewa.org on 27 th February 2013.

\section{RESUME:}

Objectif : Dans la littérature, les galles observées sur les feuilles et les tiges des Eucalyptus sont attribuées à Leptocybe invasa (guêpe à galles) et aux champignons du genre Alternaria (A. tenuis). Dans cette étude, nous avons essayé de préciser si l'isolement des Alternaria à partir des symptômes observés peut induire le développement des galles sur les feuilles des plantes d'Eucalyptus sp.

Méthodologie et résultats : Sur milieu Potato Sucrose Agar, des isolements ont été effectués à partir des galles observées sur les feuilles d'Eucalyptus. Pour l'étude du pouvoir pathogène, des inoculations artificielles ont été réalisées à l'aide d'Alternaria tenuis sur les différentes espèces d'Eucalyptus.

Les résultats obtenus des isolements réalisés ont montré la présence d'Alternaria tenuis, Les lésions foliaires ainsi que les galles des feuilles âgées peuvent héberger Alternaria. Par contre, les isolements étaient négatifs à partir des galles jeunes de couleur rouge. Alternaria tenuis, isolé des lésions infectées, induit, après inoculation des plants d'Eucalyptus, la formation des lésions foliaires. Les indices foliaires varient entre 7,5 et 17,5\%. Toutes les lésions développées sont sporulantes. Le développement des galles n'a pas été observé.

Conclusion et application de résultats: Les galles observées sur les feuilles et les pétioles des plants d'Eucalyptus ne sont pas induits par les Alternaria tenuis. Par contre, les représentants de ces champignons sont capables d'induire des lésions sur les feuilles des plants d'Eucalyptus.

Mots clés: Eucalyptus, galles, Alternaria, Postulat de Koch.

\section{SUMMARY:}

Objective: In the literature, the observed galls on the leaves and on the stems of Eucalyptus were attributed to Leptocybe invasa (gall wasp) and to the fungi of the Alternaria genus (A. tenuis). In this study, we have attempted to specify if the isolation of Alternaria from the observed symptoms may lead to the development of galls on the leaves of Eucalyptus sp. plants.

Methodology and results: On Potato Sucrose Agar, isolations were done from the observed galls on Eucalyptus leaves. For the study of pathogenicity, artificial inoculations were performed with Alternaria tenuis on different species of Eucalyptus. The results of isolations showed the presence of Alternaria tenuis, foliar lesions and galls of older leaves can host Alternaria. However, the isolation was negative for red young gall. The inoculation of Eucalyptus seedlings with Alternaria tenuis isolated from infected leaves induces the formation of leaf 
lesions. The leaf area indices vary between 7.5 and $17.5 \%$. All the developed lesions were sporulating. The development of galls was not observed.

Conclusion and Application of results: Observed galls on the leaves and petioles of plants of Eucalyptus were not induced by Alternaria tenuis. On the other side, representatives of these fungi was able to induce lesions on leaves of Eucalyptus seedlings

Key words: Eucalyptus, galls, Alternaria, Koch's postulate.

\section{INTRODUCTION}

L'extension rapide des Eucalyptus, famille des Myrtaceae (Duke, 1984), à travers le monde a entraînée divers problèmes phytosanitaires (Lanier, 1986). Les plantations d'Eucalyptus. souffrent de divers attaques d'insectes et de champignons qui se manifestent sur tiges et feuilles par différents types de symptômes Certains parasites fongiques foliaires (Mycosphaerella sp., Phaeophora sp., Cryptosporiopsis sp. et Clindorcladium sp.) ont un impact sur le rendement des espèces d'Eucalyptus de l'Asie tropical (Old et al., 2003 ; Barber, 2004).Des galles ont été observés sur les tiges, pétioles et feuilles des Eucalyptus qui se développent dans divers coins du monde : Malaisie, Indonésie, New Zélande, Australie, Afrique du sud, Chine, Egypte et Colombie (Gryzenhout et al., 2004; Jurkis 2005 ; Staden et al., 2004 ; Cortinas et al., 2006 ; Khattab, 2005 ; Dick et al., 2006 ; Gryzenhout et al., 2006 ; Dhahri et al., 2010). Leptocybe invasa, guêpe à galles originaire de l'Australie, est le principal ravageur des jeunes arbres et plantules des espèces d'Eucalyptus (Eucalyptus botryoides, E. bridgesiana, E. camaldulensis, E. globulus, E. gunii, E. grandis, E. robusta, E. saligna, E. tereticornis et E. viminalis) se développant à travers le bassin méditerranéen, le Moyen Orient (Sánchez, 2003 ;

\section{MATERIEL ET METHODES}

Des prospections réalisées dans la forêt de la Mamora (Nord ouest du Maroc), au cours du printemps-été 2010, ont permis de collecter des feuilles et des tiges jeunes d'Eucalyptus spp. présentant des galles et des lésions foliaires nécrotiques de différentes formes et couleurs. Pour vérifier la présence des Alternaria au niveau des galles, Abd-allah Salih et al. 2008 ont effectué des coupes histologiques au niveau des feuilles présentant ces types de symptômes. Les
Protasov et al.,2007), l'Afrique (Mendel, 2007), l'Asie (Doganlar, 2005 ; Hesami et al., 2005) l'Europe (Bella et al., 2002 ; Sánchez, 2003 ; Branco et al., 2009 ; EPPO, 2006), l'Amérique latine, et l'Afrique du Nord y compris le Maroc (EPPO, 2006 ; Dhahri et al., 2010). Les femelles de Leptocybe invasa produisent des galles avec des trous de sortie sur la nervure médiane des feuilles, des pétioles et des tiges. La forme des males pour cette espèces est inconnu (Mendel et al., 2004). Ces guêpes sont des inducteurs de galle et causent des dommages principalement sur les plantes d'Eucalyptus dans les pépinières et les jeunes plantations (Dhahri et al., 2010).Récemment, une étude effectuée par Abdallah Salih et Salih (2008) en Arabie Saoudite province Al-Taif a montré que les Alternaria sp. (Alternaria tenuis) peuvent être isolés à partir des même galles que ceux causés par l'insecte Cleptocybe invasa. Cependant, ces auteurs n'ont réalisés aucune inoculation artificielle des plantes d'Eucalyptus afin de vérifier le postulat de Koch. Le rapprochement des ces deux séries d'information nous a conduit à nous demander si l'isolement de l'Alternaria à partir de ces types de symptôme peut induire le développement des galles sur les feuilles et les tiges des jeunes plants d'Eucalyptus.

coupes sont déposées d'abord dans l'eau de javel pendant $15 \mathrm{~min}$, rincées ensuite dans l'eau, puis déposées pendant $15 \mathrm{~min}$ dans le carmin vert pour éliminer la chlorophylle. Les coupes colorées sont observées ensuite sous microscope. Pour l'isolement des Alternaria, les tiges et les feuilles, présentant différents types de symptômes (galles et lésions) sont abondamment lavées à l'eau courante, découpées en fragments puis placées stérilement dans des boîtes de Petri contenant 
trois rondelles de papier filtre (buvard) préalablement stérilisées puis humidifiées à l'eau distillée stérile. Les boîtes sont ensuite incubées sous lumière continue. Après 48 heures, les fragments ont été examinés sous microscope optique afin de noter la présence des fructifications des champignons. Les conidies détectées ont été transférées aseptiquement une à une sous microscope, à l'aide d'un capillaire en verre étiré, préalablement stérilisé à la flamme et refroidi dans le milieu. Ces conodies ont été déposées et étalées à la surface d'un milieu gélosé (15 g d'Agar-agar, $1000 \mathrm{ml}$ d'eau distillée), puis transférées à l'aide d'une aiguille stérilisée à la surface du milieu Potato Sucrose Agar (P.S.A). (200 $\mathrm{g}$ de pomme de terre, $20 \mathrm{~g}$ de saccharose, $15 \mathrm{~g}$ d'Agar-agar, $1000 \mathrm{ml}$ d'eau distillée). Les repiquages consécutifs accompagnés d'observations microscopiques ont permis d'obtenir des cultures pures d'Alternaria tenuis. Le postulat de Koch a été vérifié par inoculation de différentes espèces d'Eucalyptus: $E$. camadulensis, E. gomphocephala, E. grandis et des lignées nommées $419,579,583$, taille de 20 $\mathrm{cm}$, cultivés sous serre dans des pots de 16 à 20 $\mathrm{cm}$ de diamètre remplie de sable de la Mamora et de la tourbe (50/50). L'inoculum est préparé à partir des cultures d'Alternaria tenuis, cultivés sur milieu P.S.A. Ces cultures ont été incubées sous lumière continue à $25^{\circ} \mathrm{C}$. Après 7 jours, la surface chargée de conidies a été immergée avec de l'eau distillée stérile ensuite raclée à l'aide d'une spatule métallique stérile. La suspension ainsi obtenue a été filtrée à travers une mousseline afin de séparer les conidies des fragments mycéliens, puis diluée avec de l'eau distillée contenant $0,05 \%$ de Tween 20 et $5 \%$ de gélatine de façon à obtenir une concentration finale de $10^{5}$ conidies/ml. Les jeunes plants d'Eucalyptus sont inoculés par pulvérisation d'une suspension conidienne ajustée à une concentration finale de $10^{5}$ conidies $\mathrm{ml}^{-1}$ avec de l'eau distillée stérile contenant $0,05 \%$ de tween 20 et $0,5 \%$ de gélatine. En revanche, les plants témoins ont été inoculés par l'eau distillée stérile additionnée également de $0,05 \%$ de Tween 20 et $5 \%$ de gélatine. Tous les plants ont été mis sous une housse de plastique noir afin de maintenir une humidité proche de la saturation. Trois jours après les plants ont été ensuite placés sous serre afin de favoriser l'infection et le développement des symptômes. La notation des résultats est faite 7 jours après l'inoculation. La sévérité de la maladie est déterminée par le pourcentage de la surface foliaire malade (S.F.M) estimée d'après l'échelle de notation élaboré au laboratoire inspiré de l'échelle de Notteghem et al. (1980) (Figure 1 et Tableau I). Toutes les lésions formées après inoculation sont sporulantes. La sporulation est estimée par le nombre moyen de conidies produites par unité de surface de lésion $\left(\mathrm{cm}^{2}\right)$ (Hill et Nelson, 1983).

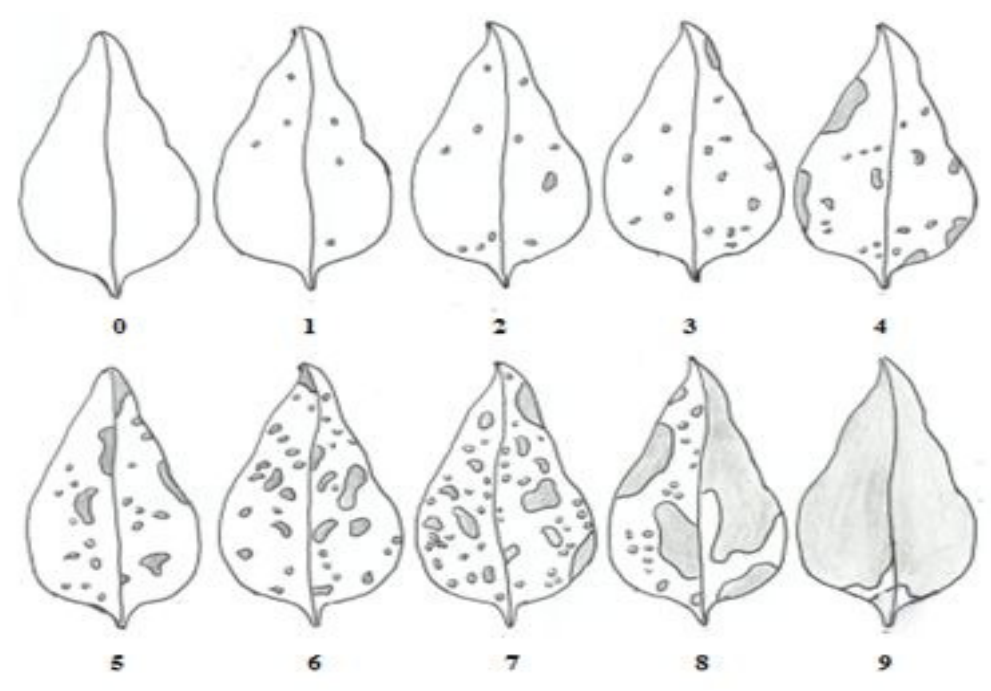

Figure 1: Echelle de notation du pourcentage de surface 
Tableau1 : Echelle de notation de Notteghem et al. (1980).

\begin{tabular}{|c|c|c|c|c|c|c|c|c|c|}
\hline Note & 1 & 2 & 3 & 4 & 5 & 6 & 7 & 8 & 9 \\
\hline$\%$ SFM & 0,05 & 0,5 & 1,5 & 3,5 & 7,5 & 17,5 & 37,5 & 62,5 & 87,5 \\
\hline
\end{tabular}

Le réisolement de l'Alternaria tenuis à partir des différentes parties des plantes inoculées a été té réalisé 20 jours après inoculation. La sporulation

\section{RESULTATS ET DISCUSSION}

Les observations effectuées sur terrain ont révélé que $80 \%$ à $90 \%$ des feuilles et des jeunes tiges d'Eucalyptus spp. Présentent des galles avec un sur hôte est estimée par le nombre moyen de conidies produites par unité de surface de lésion $\left(\mathrm{cm}^{2}\right)$ (Hill et Nelson, 1983).

pore de sortie sur la nervure médiane des feuilles. Ces galles sont de couleur rouge noirâtre (Figure $2 \mathrm{~A}$ à $2 \mathrm{C})$.
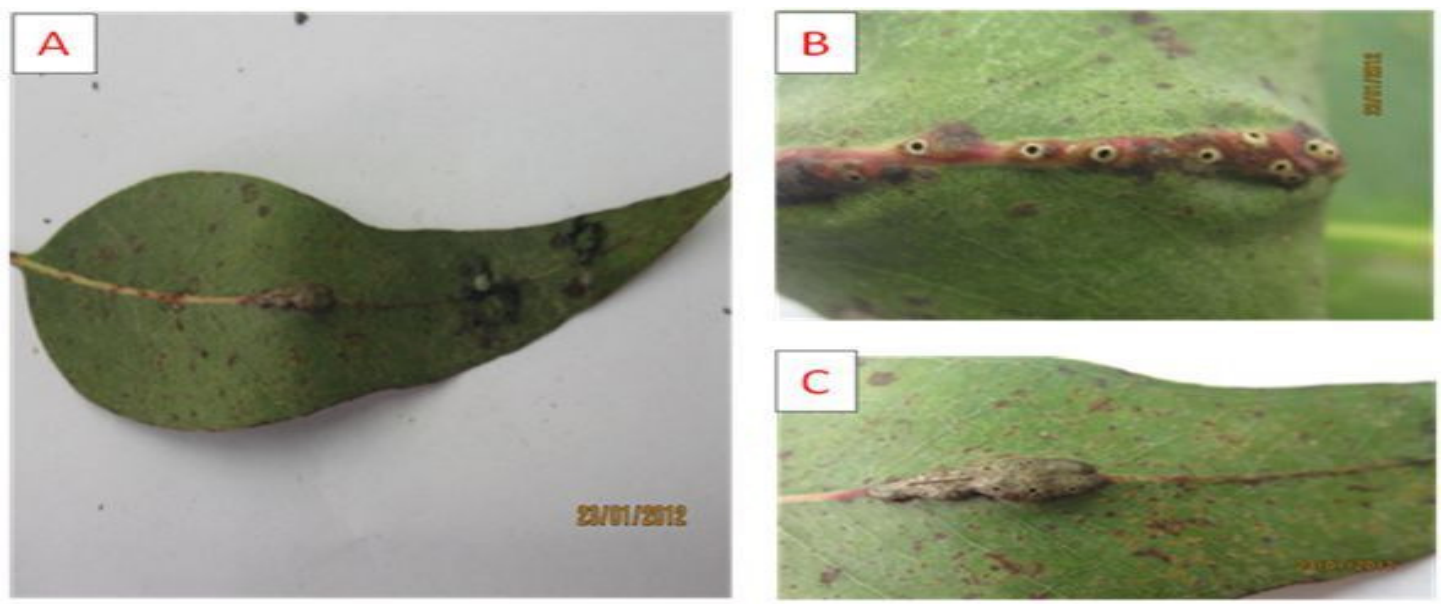

Figure 2: Feuilles d'Exucalyptsus spp. présentant des galles avec un pore de sortie sur la nervure médiane des feuilles ( $A$ à $C$ ).

En plus des galles, des lésions foliaires de 1 à 2 $\mathrm{cm}$ ont été rencontrées sur les feuilles jeunes. Ces lésions, rondes et de couleur noirâtre, peuvent fusionner avec le temps et les feuilles se nécrosent et se dessèchent (Figure $3 \mathrm{~A}$ et $3 \mathrm{~B}$ ).

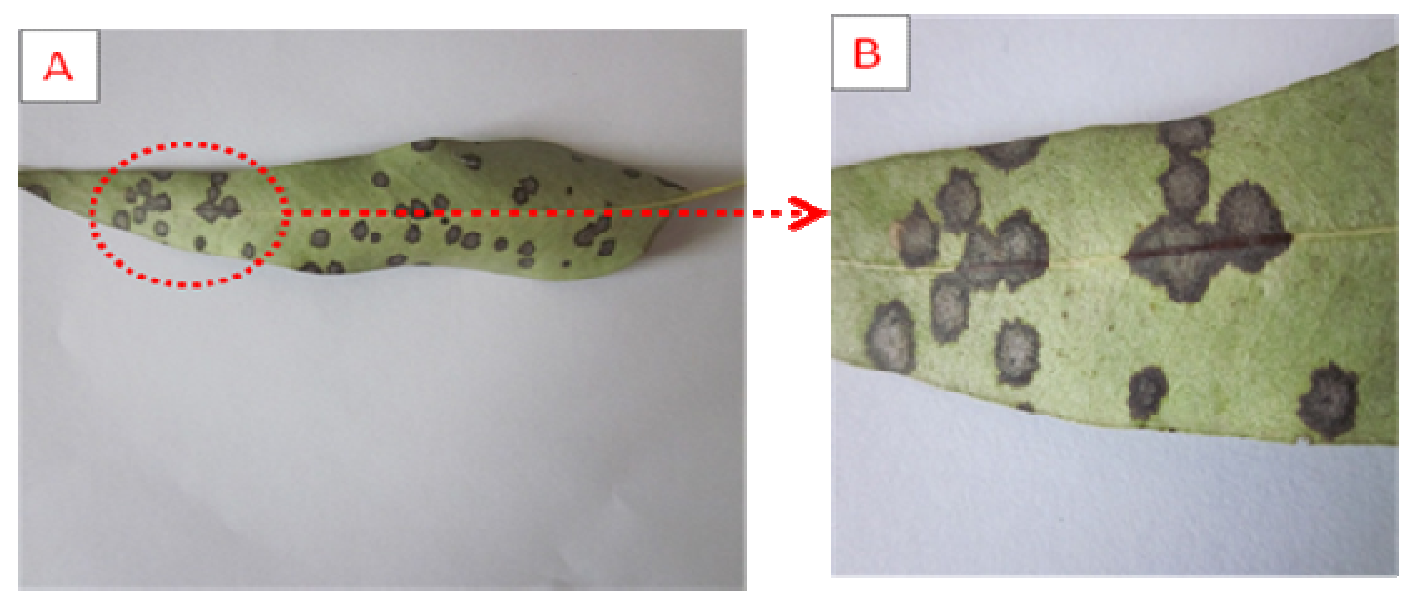

Figure 3: Différentes lésions foliaires rencontrées sur les feuilles d'Extalyptus (A et $\mathrm{B}$ ). 

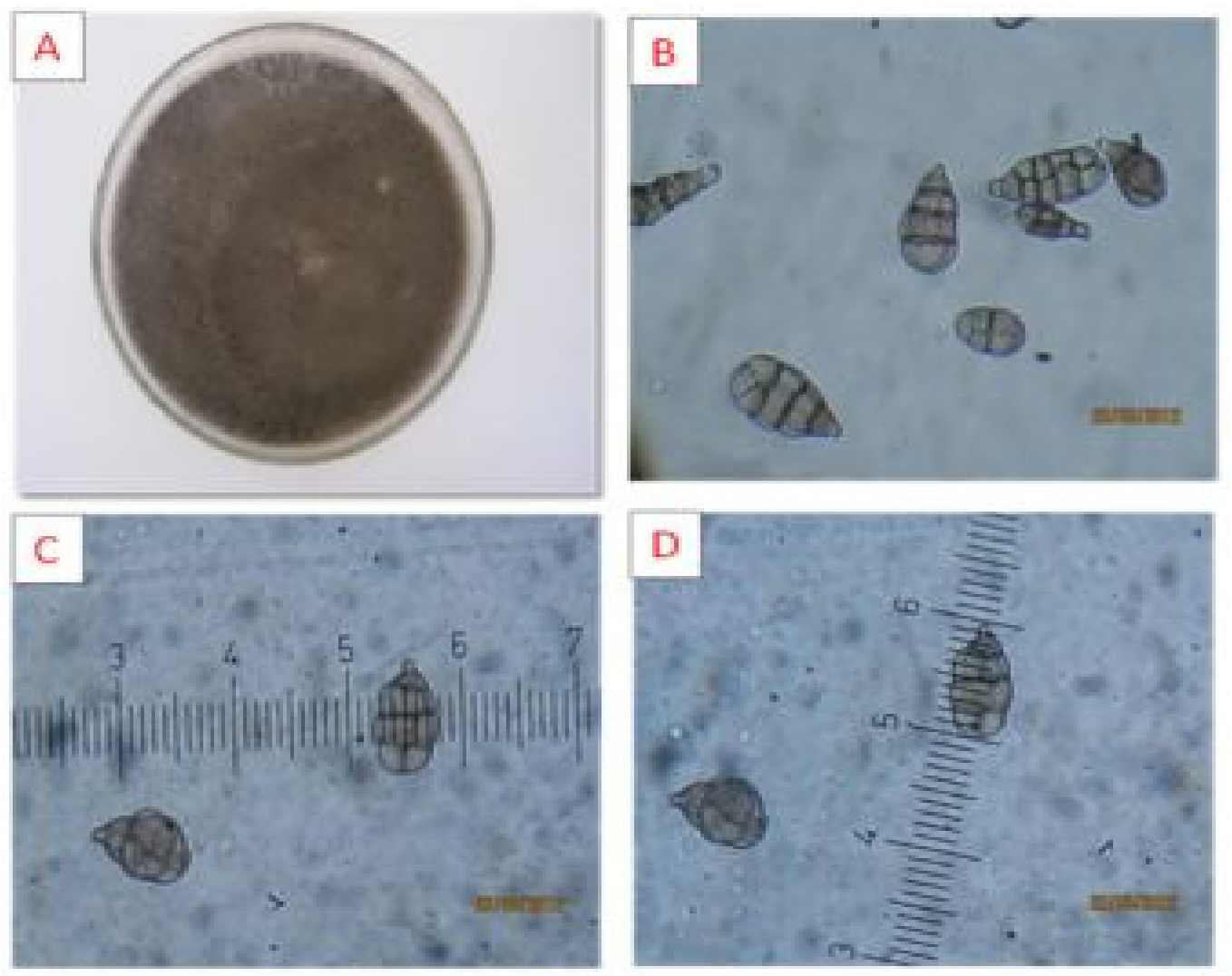

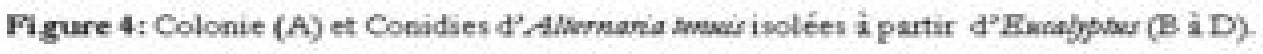

Les lésions foliaires ainsi que les galles des feuilles âgées peuvent héberger Alternaria. Par contre, les isolements étaient négatifs à partir des galles jeunes de couleur rouge, mais positif à partir des galles âgés L'espèce identifiée est Alternaria tenuis. Sur milieu de culture (PSA), Alternaria tenuis, isolé à partir des lésions foliaires et des galles âgées, présente un mycélium aérien cotonneux bien développé de couleur grisolivâtre. La culture montre des secteurs blanchâtres stériles et des secteurs de couleur gris foncé riches en conidies (figure 4A). Les conidiophores sont solitaire ou en petits groupes de cinq, de forme cylindriques, droites ou sinueuses, genouillés, 23-70 x 4-6 $\mu \mathrm{m}$, 1-6cloisonnées, couleur brune. Les conidies sont piriformes à ovoïde. Chaque conidie est formée de 3 à 5 cloisons transversales de couleur brune, certains avec un bec, mesurant 20-30 $\mu \mathrm{m} \times 12-$ $14 \mu \mathrm{m}$ (Figure 4B à 4D) Cette description est identique à celle donnée par Hennings (1902).

Les lésions commencent à se développer sept jours après inoculation des plants d'Eucalyptus, elles sont rondes de couleur brune noirâtre, de diamètres variables de 1 à $3 \mathrm{~cm}$ apparaissant au centre de la feuille ainsi qu'aux périphéries et fusionnent avec le temps et les feuilles se nécrosent et dessèchent (Figure 5A à 5D). 

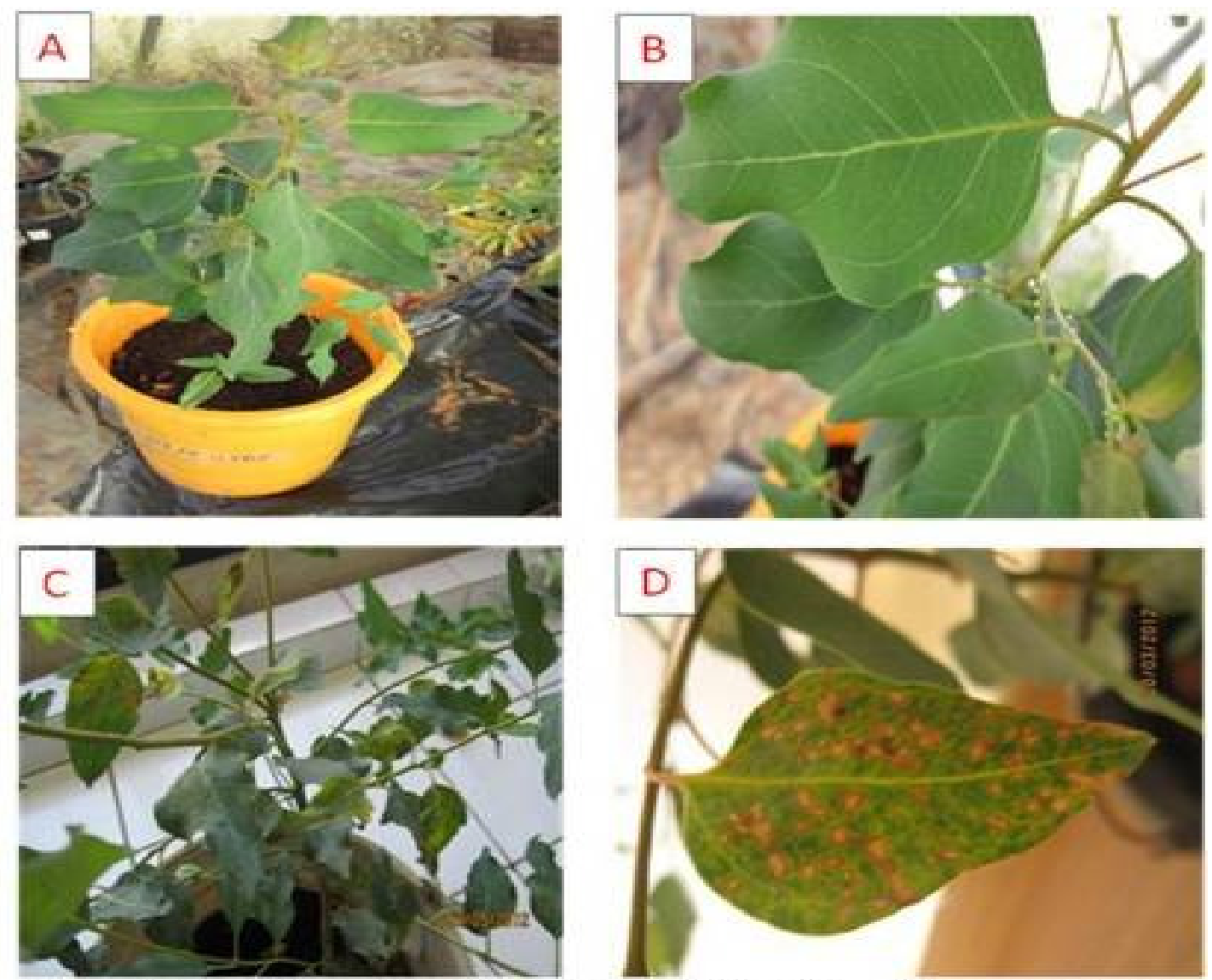

Figure 5: Plants témoins ( $\mathrm{A}$ et $\mathrm{B}$ ), plant inoculé (C) et lésions foliaires développées sur les feuilles d'Eucalpotsis inoculées par une suspension sporale d'Athernaria tersas (D).

Alternaria tenuis altère la surface foliaire des plants d'Eucalyptus inoculés. E. gomphocephala et $E$. grandis sont les plus sensibles, les indices foliaires atteignent $87,5 \%, 20$ jours après inoculation des plants. Par contre, les indices foliaires varient entre 7,5 et $17,5 \%$ chez les plants d'E. Camadulensis, 419, 579 et 583 inoculées. Toutes les lésions formées après inoculation sont sporulantes. Le nombre de conidies formées sur les lésions est de l'ordre de 4,7 $\times 10 \quad 5$ conidies $/ \mathrm{cm}^{2}$. Des études réalisées par Gibson (1975), Mittal et Sharma (1983), Rattan et Chohan (1984) ont montré $A$. tenuis, infectent est un parasite foliaire des Eucalyptus. D'autres études effectuées en Angleterre, en Australie (Fisher et al., 1993) et en Afrique du sud (Crous et al., 1987) ont rapporté le même constat. Les résultats obtenus montrent qu'Alternaria n'est pas l'agent causal des galles observés sur la nervure médiane des feuilles, mais ce champignon est responsable des lésions foliaires. Les spores d'Alternaria libérées de ces lésions peuvent profiter des pores de la surface des galles pour coloniser les parties internes des galles. L'Alternaria, observé par Abd-allah Salih et Salih (2008), au niveau des coupes histologiques réalisées au niveau des galles, ne représente qu'une colonisation ultérieure des galles déjà développées sur les feuilles ou les pétioles. Des études on montré que les galles observés sur les feuilles d'Eucalyptus sont causées par des attaque d'insecte qui causent des blessures favorisant ainsi la pénétration de divers agent pathogènes (HECFLCD, 2009). Une autre étude a été réalisée en Afrique du sud (Crous et al., 1987) sur les maladies foliaires du feuillage d'Eucalyptus ont montrée une diversité fongique dont fairt part des agents fongiques notamment 
Alternaria tenuis (Gibson, 1975 ; Mittal et Sharma, 1983 ; Rattan et Chohan, 1984).

En Espagne, une étude portant sur la diversité des espèces fongiques touchant les feuilles jeunes et adultes d'Eucalyptus Globulus, a montré que les Alternaria sont parmi les pathogènes foliaires des feuilles jeunes et adultes d'Eucalyptus globulus touchées par Mycosphaerella (Márquez et al., 2010). Ces auteurs ont isolé également des lésions foliaies différentes espèces fongiques, parmi les plus

\section{CONCLUSION}

Cette étude montre que les galles observées sur les feuilles et les pétioles des plants d'Eucalyptus ne sont pas induits par les Alternaria, Par contre, les représentants de ces champignons sont capables d'induire des lésions sur les feuilles des plants d'Eucalyptus. Mais les galles développées

\section{REFERENCES}

Abd-Allah S. \& Salih O., 2008. First report for Alternaria $\mathrm{sp}$. Infection of Eucalyptus globulus in Al-Taif province at kingdom of Saudi Arabia. Saudi J. of Bio. Sci. 15(2): 231-236.

Barber P.A., 2004. Forest Pathology: The threat of disease to plantation forests in Indonesia. Plant Pathology Journal, 3 (2). pp. 97-104.

Bella S. \& LO Verde G., 2002. Presenza nell'Italia Continentale e in Sicilia di Ophelimus prope eucalypti (Gahan) e Aprostocetus sp., galligeni degli Eucalipti (Hymenoptera Eulophidae). Naturalista sicil., 26: 191-197.

Branco M., Boavida C., Durand N, Franco J.C. \& Mendel Z., 2009. Presence of the Eucalyptus gall wasp Ophelimus maskelli and its parasitoid Closterocerus chamaeleon in Portugal: First record, geographic distribution and host preference. Phytoparasitica, 37: 51-54.

Cortinas M.N., Burgess T., Dell D., Xu D., Crous P.W., Wingfield B.D. \& Wingfield M.J., 2006. First record of Colletogloeopsis zuluense comb. nov., causing a stem canker of Eucalyptus in Cina. Mycological Research 110: 229-236.

Crous P.W, Knox-Davies P.S \& Wingfield M.J., 1987. A list of Eucalyptus leaf fungi and their Potential Importance to South African Forestry. South African Forestry African Forestry JournalNo.149. importantes ont peut citer Hormonema sp., Valsa fabianae, Readeriella dimorphospora , Neofusicoccum, Teratosphaeria molleriana, Mycosphaerella fori (=Pseudocercospora fori), Alternaria spp., Cladosporium spp., et Penicillium spp. En Angleterre et en Australie, une étude comparative des champignons endophytes qui touchent les feuilles, le xylème et l'écorce des Eucalyptus a permis l'isolement d'une diversité fongique comprenant l'agent pathogène Alternaria sp. (Fisher et al., 1993).

peuvent être colonisées après par les conidies d'Alternaria issues des lésions foliaires. Les pores des galles peuvent constituer des zones de pénétrations et d'installation de ces champignons.

Dhahri S. \& Lahbib M.B.J., 2010. First Record of Leptocybe invasa and Ophelimus maskelli Eucalyptus Gall Wasps in Tunisia. Tunisian Journal of Plant Protection Vol. 5, No. 2, 229234.

Dick M., Dobbie K., Cooke D. \& Brasier C., 2006. Phytophthora capitosa sp. Nov. and P. flaxa sp. Nov. causing crown dieback of Eucalyptus in New Zealand. Mycological Research 110: 393 404.

Doganlar O., 2005. Occurrence of Leptocybe invasa Fisher \& La Salle, (Hymenoptera: Chalcidoidea) on Eucalyptus camaldulensis in Turkey, with a description of the male sex. Zool.Middle East 35: 112-114.

Duke J.A., 1984. Borderline herbs. CRC Press. Boca raton, Florida, USA.

E.P.P.O., 2006. First report of two new eucalyptus pests in the south of France: Ophelimus maskelli and Leptocybe invasa. EPPO Reporting Service 9: 9.

E.P.P.O., 2006. Addition of Leptocybe invasa to the alert list. EPPO Reporting Service 9: 10.

Fisher P.J., Petrini O., \& Sutton B.C., 1993. A comparative study of fungal endophytes in leaves xylem and bark of Eucalyptus in Australia and England. Sydowia 45(2): 338-345.

Gibson I.A.S, 1975. Diseases of Forest Trees Widely Planted as exotics in the Tropics and Southern 
Hemisphere. Part I. Commonwealth Mycological Institute, Kew.

Gryzenhout M., Myburg, H., Van der Merwe, N. A., Wingfield, B. D. \& Wingfield, M. J. 2004. Chrysoporthe, a new genus to accommodate Cryphonectria cubensis. Studies in Mycology 50: 119-142.

Gryzenhout M., Rodas C.A., Portales J.M., Clegg P., Wingfield B.D., Wingfield M., 2006. Novel hosts of the Eucalyptus canker pathogen Chrysoporthe cubensis and a new Chrysoporthe species from Colombia. Mycological Research 110: 833-845.

Jurkis V., 2005. Eucalyptus decline in Australia and a general concept of tree decline and dieback. Forest Ecology and Management. 215: 1 20.

HECFLCD, 2009. Haut commissariats aux Eaux et Foret et a la Lutte contre la Désrtification.www. eauetforets. gov.ma/fr/index.aspx.?

Hennigs J., 1902. Macrosporium puttemansii 41: 118. 1902. = Alternaria puttemansii (Hennings) Joly, Le Genre Alternaria, p. 213. 1964.

Hesami S., Alemansoor H. \& Seyedebrahimi S., 2005. Report of Leptocybe invasa (Hym.: Eulophidae), gall wasp of Eucalyptus camaldulensis with notes on biology in Shiraz vicinity. J. Entomol. Soc. Iran 24: 99-108.

Hill J.P. \& Nelson R.R., 1983. - Genetic control of two parasitic fitness attributes of Helminthosporium maydis race T. - Phytopathology, 73(3), 455457.

Khattab H., \& Khattab I., 2005. Responses of Eucalyptus trees to the insect feeding (Gall forming Psyllid). Inter.J of Agri. And Bio. 7: 979-984.

Lanier L., 1986. Maladies de l'Eucalyptus. Bullt. OEPP/EPPOB 16, PP: 255-263.

Márquez S.S., Bills G.F. \& Zabalgogeazcoa I., 2010. Fungal species diversity in juvenile and adult leaves of Eucalyptus globulus from plantations affected by Mycosphaerella leaf disease. Annals of Applied Biology.

Mendel Z., Protasov A., Fisher N. \& La Salle J., 2004. Taxonomy and biology of Leptocybe invasa gen. \& sp. n. (Hymenoptera: Eulophidae), an invasive gall inducer on Eucalyptus. Aust. J. Entomol. 43: 51-63.

Mendel Z., Protasov A., Blumberg D., Brand D., Saphir N., Madar Z. \& La Salle J., 2007. Release and recovery of parasitoids of the Eucalyptus gall wasp Ophelimus maskelli in Israel. Phytoparasitica 35: 330-332.

Mittal R.k. \& Sharma M.R., 1983. Two new fungi in Eucalyptus nurseries in India. Indian Journal of Mycology and Plant Pathology 12: 69. (Review of Plant Pathology 62: 434, abst. 4778).

Notteghem J.L., Andriatompo G.M., Chatel M. \& Dechanet R., 1980. Techniques utilisées pour la sélection de variétés de riz possédant la résistance horizontale à la pyriculariose. - Ann. Phytopathol., 12: 199-266.

Old K.M., Wingfield M.J. \& Yuan Z.Q., 2003. A Manual of Diseases of Eucalyptus in South-East Asia. Center for International Forestry Research, Bogor.

Protasov A., Blumberg D., Brand D., La Salle J. \& Mendel Z., 2007. Biological control of the gall wasp Ophelimus maskelli (Ashmead): Taxonomy and biology of the parasitoid species Closterocerus chamaeleon (Girault), with information on its establishment in Israel. Biological Control, 42: 196-206.

Rattan G.S. \& Chohan J.S, 1984. Some common diseases of Eucalyptus in the Punjab. Indian Journal of Mycology and Plant Pathology 13:90. (Review of Plant Pathology 64: 400, abstr, 3977).

Sánchez I., 2003. Descubiertas dos nuevas plagas del eucalipto en España. Quercus, 214: 32-33.

Staden V., Erasmus B., Roux J., Wingfield MJ. \& Jaarsveld A. 2004. Modeling the spatial distribution of two important South African plantation Foresty pathogens. Forest Ecology and Management. 187: 61-73. 